\title{
Improved IHS Pan-Sharpening Method Based on Adaptive Injection of À trous Wavelet Decomposition
}

\author{
Saber Noroozpour Lari*1 ${ }^{1}$, Mehran Yazdi ${ }^{2}$ \\ ${ }^{1}$ School of Electrical and Computer Engineering, Shiraz University, Shiraz, Iran \\ ${ }^{2}$ School of Electrical and Computer Engineering, Shiraz University, Shiraz, Iran \\ 's.noroozpour@gmail.com, 2yazdi@shirazu.ac.ir
}

\begin{abstract}
The goal of pan-sharpening is to increase both spatial and spectral resolution of multispectral images. Intensity-hue-saturation (IHS) is one of widely efficient image fusion methods used in recent years. The drawback of IHS is spectral distortion in its results which can be improved by use of wavelet decomposition in IHS-based pansharpening methods. Employing Wavelet transforms enhances the resolution of Multispectral (MS) images while maintaining the spectral properties. This paper presents an adaptive IHS-based fusion using "à trous" wavelet (ATW) decomposition based on injecting weighted high frequency components of high spatial panchromatic (PAN) image obtained through à trous decomposition into resampled version of the MS images. Furthermore, the parameters used in the proposed algorithm are optimized through the genetic and Teaching-Learning algorithms. Finally, the proposed method is evaluated using the IKONOS and Landsat ETM+ images and compared to the other conventional methods to confirm its superiority.
\end{abstract}

Keywords: à trous wavelet; genetic algorithm; image fusion; intensity-hue-saturation; pan-sharpening

\section{Introduction}

Optical remote sensing sensors such as IKONOS, QuickBird, and Landsat ETM+ provide two types of images, a high spatial resolution panchromatic (PAN) image with low spectral quality and low spatial resolution multispectral (MS) image with higher spectral resolution than PAN. Remote sensing applications are severely widespread for which it is necessary to have high spatial and spectral resolutions to get the best results. Hereby, the fusion of MS and PAN images, known as pan-sharpening, is a technique which helps us to have a fused image with properties of high spectral MS images and high spatial quality of PAN images.

There are varieties of fusion methods in pan-sharpening branch [1,2]. Three types of pan-sharpening methods have been introduced in recent years such as component substitution (CS) based methods [3], relative spectral contribution based ones and multi resolution analysis (MRA) based pan-sharpening [4]. Some methods such as IHS $[1,5]$ and principal component analysis (PCA) $[6,7]$ are based on component substitution (CS), on the other hand, methods such as intensity modulation [8] and Brovey [9] are based on relative spectral contribution. These techniques are very popular because they are fast and efficient; however, they do not preserve spectral information of MS data. Even though, like the other CS techniques, IHS-based method enhances the spatial resolution, it simultaneously produces spectral distortion. Several researchers have proposed methods

*Corresponding Author 
to improve the spectral distortion of IHS-based fusion methods [10-12]. One of the popular approaches for minimizing spectral distortion is based on the extraction of high frequency spatial information from the PAN data and then injection of them to MS images. These methods, usually called Multi resolution Analysis-based methods, are initiated by the high-pass filtering method [7] and then continued with algorithms such as discrete wavelet transform[4, 9, 13, 14], Laplacian pyramid algorithms [15] and à trous wavelet (ATW) transform $[4,16,17]$. MRA-based methods obviate the spectral distortion problem, but the spatial quality of its result is lower than that of both CS and relative spectral contribution based methods. In addition, the mixture of CS and MRA techniques can provide images with both spatial and spectral quality. Therefore, adaption of the spatial details injection can more improve quality of the results [18].

In this paper, we employ ATW decomposition for extraction of the high frequency spatial details of PAN image and intensity components of MS images, and then we add the spatial details of PAN image to intensity components. In order to improve the quality of fusion and to preserve the spectral information of MS images, we adapt the spatial details injection by a set of appropriate gains. Two optimization algorithms determine these gains in order to have high spatial and spectral quality.

This paper is organized as follows. Section 2 presents some recent pan-sharpening methods. Genetic and Teaching-Learning optimization methodology is introduced in Section 3. Our proposed method is described in Section 4 and the experimental results and comparisons with some well-known methods are provided in Section 5. Section 6 concludes the paper.

\section{Overview of Some Pan-sharpening Methods}

\subsection{IHS Fusion based Method}

The IHS transform is a widely used merger in image fusion processes. The widespread use of IHS fusion method is based on its ability to separate the spectral and spatial information of an RGB image[19]. This method is always applied to an RGB composite and gives us three new components as results, i.e. Intensity, Hue, and Saturation components. Intensity component contains most of the spatial information of RGB image; Hue and Saturation components comprise spectral information.

The IHS technique is usually composed of four steps.

Step 1: Transforming the RGB image into the IHS components.

Step 2: Matching the histogram of the PAN image to the intensity component histogram. Histogram matching is performed to stretch the spectral range of PAN and to match the histogram of PAN to the histogram of intensity component. This is done in order to minimize spectral distortion of the fused image.

Step 3: Replacing the intensity component by PAN image.

Step 4: Applying the inverse transform and obtaining RGB image with the spatial details of the PAN image.

Although the IHS enhancement is adequate enough for some purposes, due to undesirable colour distribution in its results, variety of researchers have been conducted to improve it. 


\subsection{Generalized IHS}

To overcome the limitation of IHS when there are more than three bands of MS images for being fused with a PAN image, as in the cases of IKONOS, QuickBird, or Landsat ETM+ data fusion, Tu et al. proposed a generalized IHS (GIHS) transform. In this modified IHS method, NIR band is involved in fusion. In GIHS method, new bands are obtained from the next equation.

$$
\hat{\boldsymbol{B}}_{j}=\tilde{\boldsymbol{B}}_{j}+\boldsymbol{D}
$$

Where $\tilde{\mathbf{B}}_{j}$ and $\hat{\mathbf{B}}_{j}$ refer to MS image up-sampled to the PAN scale and fusion products, respectively. D presents the spatial details of PAN image as follows.

$$
\boldsymbol{D}=\boldsymbol{P}-\frac{1}{N} \sum_{j=1}^{N} \tilde{\boldsymbol{B}}_{j}
$$

The index $j$ denotes each band of an MS image, and $N$ is equal to the number of MS bands. $\mathbf{P}$ also represents the PAN data.

Similar to the IHS and many other fusion methods, the shortcoming of GIHS fusion method is colour distortion of its results.

\subsection{PCA}

Similar to IHS, PCA is based on the CS technique. Applying PCA leads to uncorrelated principal components by a linear combination. In the PCA-based fusion method, the first principal component (PC1) in PCA space, which carries the spatial information of the MS images, is replaced by the high spatial resolution PAN image[7, 14].

Spectral ranges of multispectral and panchromatic images in some satellite sensors are not matched. This difference in spectral ranges leads to spectral distortion in results, similar to what occurs in his method. In order to minimize spectral distortion of the fused image, before replacing PAN with PC1, the panchromatic image is histogram-matched with the first component of the results in PCA space. Inverse PCA is then performed on the PC and PAN images to obtain a fused image with spatial details of PAN image.

\subsection{Multi-resolution Analysis based Image Fusion Methods}

The wavelet image fusion produces the acceptable resolution without altering the spectral content of the image. There are two main groups of wavelet transform: continuous wavelet transforms (CWT), and discrete wavelet transforms (DWT). Approximately in all papers, DWT is used for fusion applications. This is subdivided in various approaches; decimated $[9,20,21]$ and undecimated wavelet transform algorithms $[14,22]$ are two major groups of wavelet based image fusion methods. Both of decimated and undecimated wavelet transforms decompose the image into approximation part and details. In decimated wavelet, the spatial resolution is divided in each level by power of two, whereas in undecimated algorithm the images of each level have same size. That is performed to preserve some detail information $[4,23]$.

The main idea of MRA-based pan-sharpening is based on the decomposition of both low and high spatial resolution images. Each image at each wavelet transform level has one low spatial resolution image and several detail ones. The detail parts of MS images are replaced by or added to the details of PAN. Then, the inverse multi-resolution wavelet is applied to obtain the high-resolution MS image. In this scenario, image size is often 
limited to power of two and artifacts might be produces in subsampling. To overcome these limitations, an undecimated algorithm has been proposed by Shensa et al.[16].

The ATW is an undecimated shift-invariant algorithm. Prior to the jth level decomposition, the low-pass filter in ATW decomposition is up-sampled by $2^{j}$. This is well suited to cases of image fusion where the image size is not powers of two. The ATW decomposition algorithm decomposes original image into a sequence of new images, called wavelet planes $\left(\mathbf{W}_{\mathbf{i}}\right)$, these wavelet planes can be obtained by adopting several methods described in the following. There are many possible ways of à trous and other wavelet algorithm in the MRA-based pan-sharpening methods, i.e. additive method and substitution method. In wavelet additive method details of PAN image are directly added to the MS image, but in the wavelet substitution method, the MRA is performed on both the PAN and the MS images; this is due to the fact that the aim of MRA is to incorporate the details from the PAN image into the MS image where the approximation image of the PAN image is discarded. Moreover, there are some methods that select the detail coefficients[4] from either the MS or the PAN image at each pixel location based on a selection rule.

2.4.1 Additive Wavelet Luminance Proportional (AWLP): This fusion method is based on à trous wavelet transform. In this method high resolution PAN image is decomposed into ' $n$ ' wavelet planes, whaere' $n$ ' is computed by:

$$
n=\log _{2}\left(r_{L R M} / r_{H R P}\right)
$$

Where $r_{\text {LRM }}$ and $r_{\text {HRP }}$ are the low resolution MS image spatial resolution and high resolution PAN image spatial resolution, respectively

Then the high resolution PAN detail is injected into every original MS band based on original radiance value of each bands [24] by using Eqs.(4) and (5).

$$
\begin{aligned}
& \boldsymbol{P}=\sum_{l=1}^{n} \boldsymbol{\omega}_{P_{l}}+\boldsymbol{P}_{r} \\
& \alpha_{j}=\left(\frac{\tilde{\boldsymbol{B}}_{j}}{\frac{1}{L} \sum_{j=1}^{L} \tilde{\boldsymbol{B}}_{j}}\right) \\
& \hat{\boldsymbol{B}}_{j}=\alpha_{j} \sum_{l=1}^{n} \boldsymbol{\omega}_{P_{l}}+\tilde{\boldsymbol{B}}_{j}
\end{aligned}
$$

Here wavelet planes $\boldsymbol{\omega}_{P_{l}}$ are injected to each upsampled MS image $\tilde{\mathbf{B}}_{j}$ with a coefficient $\alpha_{j}$. L is the number of MS bands.

\subsection{Generalized Laplacian Pyramid with Spectral Distortion Minimization (GLP-SDM)}

The goal of this fusion method is to obtain MS bands with same spatial resolution as PAN data based on Laplacian pyramid. In this method, firstly the Low Resolution PAN (LRP) image is produced from upsampling of downsampled original PAN data. Then, the high-pass component of PAN (HPP) obtained from difference of original PAN and LRP is weighted by $\alpha_{j}$ parameters [25] as follows: 


$$
\alpha_{\mathrm{j}}=\frac{\tilde{\boldsymbol{B}}_{\boldsymbol{j}}}{\boldsymbol{L R P}}
$$

Here $\tilde{\mathbf{B}}_{j}$ refers to MS image up-sampled to the scale of the PAN image.

Next, the weighted HPP is injecting to each $\tilde{\mathbf{B}}_{j}$ to obtain high resolution MS bands as follows:

$$
\hat{\boldsymbol{B}}_{j}=\alpha_{j} \times \boldsymbol{H P P}+\tilde{\boldsymbol{B}}_{j}
$$

Here, $\hat{\mathbf{B}}_{j}$ refers to fused images.

\section{Optimization Methodology}

\subsection{Basic Concept of Genetic Algorithm}

Genetic algorithm (GA) is a population based optimization search method inspired from the natural biological process. Each population is a chromosome considered as a possible solution for the problem. The progress in population is done in three steps, selection, cross over and mutation [26]. In the selection step the chromosome which is allowed to reproduce is chosen. Crossover dislocates the subsections of two chromosomes and in the mutation step some of genes in chromosome are changed randomly. These steps are described as follows.

3.1.1 Selection: This operator chooses the chromosomes which have the survive chance and allows them for reproduction. The chromosome with better fitness value has more probability for reproduction. The roulette wheel mechanism[27] can be used to select the chromosome as parents for the next step.

3.1.2 Crossover: The selected parent chromosomes are stored in a mating pool. Here, two of them are selected randomly for crossover. To do this, a random locus (a bit position along the chromosome) is selected randomly and the bits after this point are exchanged between these two chromosomes. The new generated chromosomes are considered as offspring. Parent chromosomes are substituted by offspring if they are defined as the better solutions.

3.1.3 Mutation: This operator flips the value of one or more bits of the offspring which are selected randomly in a chromosome. This change causes the diversity of the population is kept and restraints the premature convergence.

\subsection{Using GA for Pan-sharpening}

In this section, the use of GA on our pan-sharpening problem is presented in details. The procedure of solving this problem is described in the following steps:

Step 1: Classifying the basic information. This information is divided into two types. First type, information about the optimization algorithm including the number of initial population (number of chromosomes), the number of iterations, the mutation rate, the number of bits devoted to each control variables, and upper and lower limits corresponding to the control variables. Second type, information about the pan-sharpening problem including PAN data and MS images. 
Step 2: Generating the population

This step has two sub-steps:

Step 2-a): Generating the initial population as a set of binary bits.

$$
\begin{aligned}
& \text { initial_population }=\left[\begin{array}{c}
\boldsymbol{X}_{1} \\
\boldsymbol{X}_{2} \\
\vdots \\
\boldsymbol{X}_{\boldsymbol{n}}
\end{array}\right]_{n \times z} \quad i=1,2, \ldots ., n \\
& \boldsymbol{X}_{i}=\left[x_{i, 1}, x_{i, 2}, \ldots, x_{i, z}\right]_{1 \times z} \\
& \begin{array}{l}
z=N_{\mathrm{var}} \times N_{b i t} \\
x_{i, j}=0 \text { or } 1
\end{array} \quad i=1,2, \ldots, n \quad j=1,2, \ldots ., z
\end{aligned}
$$

Where $n$ is the number of initial population (chromosomes), $z$ is the length of each chromosome, $N_{v a r}$ is the number of control variables, and $N_{b i t}$ is the number of bits devoted to each control variable.

Step 2-b): Decoding the binary chromosomes

To this end, each chromosome is considered as a binary digit and decoded to a decimal digit. Here the population is generated in the problem search space, as follows.

$$
\begin{aligned}
& x_{i}=\frac{x_{d e c, i}}{2^{N_{b i t}-1}}\left(\max _{-} \text {var-min_var }\right)+\text { min_var } \quad i=1,2, \ldots, N_{\mathrm{var}} \\
& \boldsymbol{X}_{j}=\left[x_{1}, x_{2}, \ldots, x_{N_{\mathrm{var}}}\right]_{1 \times N_{\mathrm{var}}} \\
& \text { population }=\left[\begin{array}{c}
\boldsymbol{X}_{\boldsymbol{I}} \\
\boldsymbol{X}_{2} \\
\vdots \\
\boldsymbol{X}_{\boldsymbol{n}}
\end{array}\right]
\end{aligned}
$$

Where $x_{d e c, i}$ is a decimal value related to the $i^{\text {th }}$ control variable.

Step 3: Calculating the objective function

To do so, the QNR index, as defined in Eq.(10), is considered as an objective function.

$$
Q N R=\left(1-D_{\lambda}\right)^{\alpha}\left(1-D_{s}\right)^{\beta}
$$

Where $D_{s}$ and $D_{\lambda}$ explained in section 5.1.4, calculate the spatial and spectral distortion, respectively. $\alpha$ and $\beta$ are in the interval $[0,1]$, and the maximum value of QNR is 1 at zero value of spatial and spectral distortion.

Step 4: Selecting the Parents

In this step, the parent chromosomes are selected to generate the new population. Selection procedure is done based on the Roulette Wheel Mechanism (RWM). Since the lower fitness defines the better solution, thus, the reverse fitness is considered as the probability for RWM. The selected parents are added to the mating pool and wait for the next step. 


\section{Step 5: Crossovering the Parents}

For generating the children from the parents, first, two chromosomes are selected from the mating pool randomly. Here, the bits of parent chromosomes are substituted from the break point which is itself selected randomly. Between the parents and children chromosomes each of which produces the better solutions is defined as the new population for the next iteration.

Step 6: Mutating the new population.

To prevent the premature convergence for the population and to extend the search space, the new population obtained in the previous step is mutated. To this end, based on the mutation rate, some of the chromosome bits, which are selected randomly, are changed.

\section{Step 7: Checking the Convergence Criterion}

In this step convergence criterion is checked; if it is satisfied then the process is stopped and the best solution is selected as the response of the problem, otherwise the algorithm is returned to the step (2-b).

\subsection{Basic Concept of Teaching-Learning Algorithm}

Teaching-learning-based optimization (TLBO) algorithm is based on the influence of teacher ability on learners. Teacher usually shares his/her knowledge with learners; hence, more knowledge of teacher yields more effect on learning. If each learner has a score in final, the mean of class score increases based on the ability of teacher and the interaction of learners.

The process of this algorithm is divided into two phases; teacher phase and learner phase as follows.

3.3.1 Teacher Phase: The highly-learned person in a class (a group of learners) is considered as a teacher. A good teacher gives his/her knowledge to learners and improves their grades to some extent with respect to the capability of the learners and boosts the mean of the class. Assume $M_{i}$ is the mean of the class and $T_{i}$ is the teacher in each iteration, then we have:

$$
{\text { Difference } \_M e a n_{\mathrm{i}}}=r_{i}\left(\boldsymbol{M}_{\text {new }}-T_{\mathrm{F}} \boldsymbol{M}_{i}\right)
$$

Where TF is a teaching factor that decides the value of mean to be changed and ri is a random number between 0 and $1 . M_{\text {new }}$ is the new mean obtained from upgraded class.

Therefore, the solution can be updated according to the following equation:

$$
\boldsymbol{X}_{\text {new } 1, i}=\boldsymbol{X}_{\text {old }, i}+\text { Difference_Mean }{ }_{i}
$$

3.3.2 Learner phase: Learners improve their knowledge by two ways; by using the teacher and by interacting among themselves. A learner takes the knowledge of the other learners randomly, and the knowledge of learner is improved if the second learner has more knowledge than him/her. Learner improvement is defined as:

$$
\left\{\begin{array}{l}
\boldsymbol{X}_{\text {new }_{2}, i}=\boldsymbol{X}_{\text {old }, i}+\text { rand }_{i} \cdot\left(\boldsymbol{X}_{i}-\boldsymbol{X}_{j}\right) \text { if } f\left(\boldsymbol{X}_{i}\right)>f\left(\boldsymbol{X}_{j}\right) \\
\boldsymbol{X}_{\text {new }_{2}, i}=\boldsymbol{X}_{\text {old }, i}+\text { rand }_{i} \cdot\left(\mathbf{X}_{j}-\mathbf{X}_{i}\right) \text { if } f\left(\boldsymbol{X}_{j}\right)>f\left(\boldsymbol{X}_{i}\right)
\end{array}\right.
$$


Finally, each of $\mathbf{X}_{\text {new j,i }}(j=1,2)$ which has a better solution is selected as $\mathbf{X}_{\text {new }}$. If $\mathbf{X}_{\text {new,i }}$ defines a better solution than $\mathbf{X}_{\text {old,i }}$, it is replaced by new $\mathbf{X}$.

\subsection{Steps of TLBO}

Step 1: Import the basic data.

Step 2: Change stochastic problem into its corresponding deterministic scenarios.

Step 3: Generate the initial population, i.e. a class of learners.

$$
\begin{aligned}
& \text { Initial_Population }=\left[\begin{array}{c}
\boldsymbol{X}_{1}^{\text {total }} \\
\boldsymbol{X}_{2}^{\text {total }} \\
\vdots \\
\boldsymbol{X}_{n}^{\text {total }}
\end{array}\right]_{n \times z} \\
& \boldsymbol{X}_{l}^{\text {total }}=\left[\boldsymbol{X}_{l, 1}, \boldsymbol{X}_{l, 2}, \ldots, \boldsymbol{X}_{l, n}\right]_{(1 \times z)} l=1,2, \ldots, n \\
& \boldsymbol{X}_{\boldsymbol{l}}=\boldsymbol{r a n d} \times(\boldsymbol{m a x} \text { avar }-\boldsymbol{m i n} \text { in_var })+\boldsymbol{m i n} \text { inar }
\end{aligned}
$$

Where, $n$ is the number of initial population.

Step 4: Calculate the objective functions of each individual as in Eq.(10).

Step 5: Determine the fitness of each member of $\mathbf{X}_{l}^{\text {total }}$.

Note that, steps 4 and 5 are repeated for all members of initial population.

Step 6: Select the teacher of the class; the solution (learner) with the best fitness value (more knowledge) is considered as the teacher of the class.

Step 7: Improve the knowledge of learners; for this purpose, $\mathbf{X}_{n e w 1}$ is generated using Eq.(12) and if it gives a better solution than $\mathbf{X}_{\text {old }}$, it is replaced with $\mathbf{X}_{\text {new1 }}$. Then $\mathbf{X}_{\text {new } 2}$ is generatedusing Eq.(13). $\mathbf{X}_{\text {new2 }}$ is accepted if it reveals a better fitness value.

Step 8: Check the convergence criterion; if it is satisfied then the process is stopped and the best solution is selected as the response of the problem, otherwise the algorithm is returned to the step 6 .

\section{Improved Adaptive IHS-ATW Merger}

Original IHS fusion method provides the fused image with spectral quality of MS images when PAN and MS images are highly correlated. In the datasets conveyed from IKONOS or Landsat ETM+ satellites, the bandwidth of PAN data does not cover those of MS images and it causes spectral distortion in IHS fusion results.

To resolves this problem, researches have proposed improved IHS based on wavelet decomposition with a combinational use of IHS and wavelet [14,17]. These researchers have utilized wavelet decomposition to extract high spatial resolution of PAN image and then injected it into MS images. The joint use of IHS and wavelet helps to eliminate spectral distortion of IHS results while spatial resolution of PAN image is quite injected into fused image.

In this paper, we enhance the improved IHS approach by using an adaptive method based on ATW decomposition. We choose an optimal number of levels for decomposition based on Eq.(3) introduced in [24].

According to Eq.(3), number of levels for decomposition for IKONOS data and Landsat ETM+ are 2 and 1, respectively.

ATW is selected because of its redundant decomposition without losing information. The stages of applying our proposed method to IKONOS dataset are described as follows. 
In the $i$ th level of processing for computing $W_{i}$, the image $C_{i-1}$ and convolution kernel $\mathrm{h}_{\mathrm{i}}$ will be used. $C_{0}$ is defined as the original image, $h_{i}$ and $C_{i}$ are computed as follows.

$$
\begin{aligned}
& \boldsymbol{h}_{i}=\frac{1}{256}\left(\begin{array}{ccccc}
1 & 4 & 6 & 4 & 1 \\
4 & 16 & 24 & 16 & 4 \\
6 & 24 & 36 & 24 & 6 \\
4 & 16 & 24 & 16 & 4 \\
1 & 4 & 6 & 4 & 1
\end{array}\right) \\
& \boldsymbol{C}_{i}=\boldsymbol{C}_{i-1} \otimes \boldsymbol{h}_{i}
\end{aligned}
$$

Where $\otimes$ represents the convolution operator.

After computing $\mathbf{C}_{i}$, wavelet plane ( $\mathbf{W}_{i}$ ) can be obtained using:

$$
\boldsymbol{W}_{i}=\boldsymbol{C}_{i-1}-\boldsymbol{C}_{i}
$$

Note that in each iteration, the kernel $h_{i}$ is modified by inserting zeros between the original elements, but these values are ignored in performing the fusion process. The size of $\mathbf{h}_{i+1}$ after modification is twice the size of $\mathbf{h}_{\mathbf{i}}$. The wavelet processing is continued for $" n "$ iterations $(n=4)$. Next, the original image can be reconstructed by:

$$
\boldsymbol{I}=\sum_{i=1}^{n} \boldsymbol{W}_{i}+\boldsymbol{C}_{n}
$$

Here, $\mathbf{C}_{n}$ is an approximation image resulted by applying wavelet transform, and $\mathbf{W}_{1}$, $\ldots, \mathbf{W}_{n}$ planes are detail images.

$$
\begin{aligned}
& {\left[\begin{array}{c}
I \\
v 1 \\
v 2
\end{array}\right]=\left[\begin{array}{ccc}
1 / 3 & 1 / 3 & 1 / 3 \\
-\sqrt{2} / 6 & -\sqrt{2} / 6 & 2 \sqrt{2} / 6 \\
1 / \sqrt{2} & -1 / \sqrt{2} & 0
\end{array}\right]\left[\begin{array}{l}
R \\
G \\
B
\end{array}\right]} \\
& H=\tan ^{-1}\left(\frac{v 1}{v 2}\right) \quad \text { (b) } \quad S=\sqrt{v 1^{2}+v 2^{2}}
\end{aligned}
$$

Where $I$ shows the pixels of the intensity component image and $v 1$ and $v 2$ represent Hue $(\mathrm{H})$ and Saturation(S) images using Eqs.(19b) and (19c).

The schematic flowchart of the proposed fusion algorithm is shown in Figure.1. 


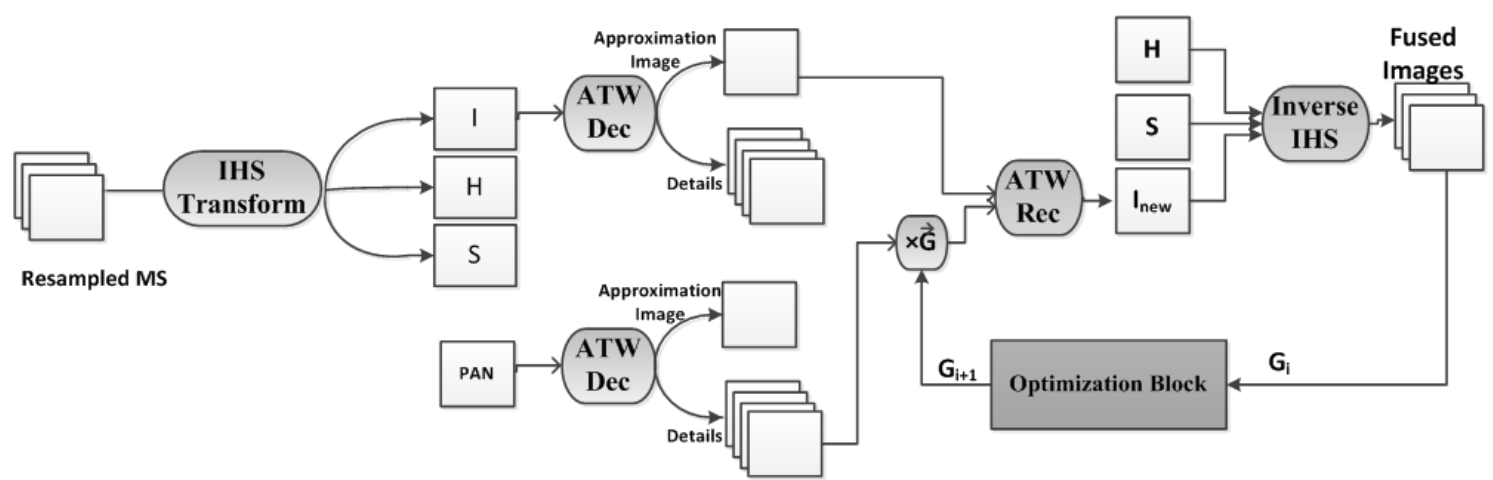

Figure 1. Schematic Procedure of the Fusion Algorithm

After registering MS and PAN images, the IHS transform is applied to the three bands of MS images (19). Then, considering (20) and (21), intensity components (I) obtained from IHS transform and PAN image (P) are decomposed into an approximation image ( $\mathbf{I}^{\mathrm{LF}}$ and $\mathbf{P}^{\mathrm{LF}}$ ) and four images with high frequency details information $\left(\mathbf{I}_{i}^{\mathrm{HF}}\right.$ and $\mathbf{P}_{i}^{\mathrm{HF}}$ ), with same resolution as PAN data.

$$
\begin{aligned}
& \boldsymbol{I}=\sum_{i=1}^{4} \boldsymbol{I}_{i}^{\mathrm{HF}}+\boldsymbol{I}^{\mathrm{LF}} \\
& \boldsymbol{P}=\sum_{i=1}^{4} \boldsymbol{P}_{i}^{\mathrm{HF}}+\boldsymbol{P}^{\mathrm{LF}}
\end{aligned}
$$

We reconstruct high frequency image of new intensity component ( $\left.\mathbf{I}_{\text {new }}\right)$ by adding details obtained from intensity component and weighted details of PAN image by use of the following equation.

$$
\begin{gathered}
\boldsymbol{I}_{\text {new }}^{\mathrm{LF}}=\boldsymbol{I}^{\mathrm{LF}} \\
\boldsymbol{I}_{\mathrm{new}_{i}}^{\mathrm{HF}}=\boldsymbol{I}_{i}^{\mathrm{HF}}+g_{i} \cdot \boldsymbol{P}_{i}^{\mathrm{HF}}
\end{gathered}
$$

Where $\mathbf{P}$ and $\mathbf{I}$ refer to PAN and Intensity component images, and $L F$ and $H F$ subscripts refer to high frequency and low frequency parts of images, respectively. Optimization gain $g_{i}$ is used to inject high frequency information of PAN image into $\mathbf{I}_{\text {new }}$, if needed. Here, GA and TLBO are employed for optimizing gains. To preserv the spectral content of MS images and have a better spatial quality in the fused images, we use two optimization algorithms to adapt the gains with minimizing fitness function. The fitness function in this work is the QNR, as defined in Eq.(10). QNR indicator consists of two indices $D_{s}$ and $D_{\lambda}$. $D_{s}$ is difference of UIQI value for inter-MS band and inter-fused band as spectral distortion and $\mathrm{D}_{\lambda}$ is the difference of UIQI value between each MS band and the PAN image at low resolution and in the high resolution as spatial distortion. If we consider these two indicators together, a fused image with spectral quality of MS images and spatial quality of PAN image can be obtained.

After parameters optimization, the reconstructed ATW is applied on $\mathbf{I}^{\mathrm{LF}}$ and $\mathbf{I n e w}_{i}^{\mathrm{HF}}$, and the new intensity component with high spatial information of PAN data is reconstructed. Then the fused images are achieved from inverse IHS transform. The fusion scheme is shown in Figure 1. 


\section{Case Studies}

In this section, some experiments have been performed on two datasets. The first dataset is IKONOS dataset with four MS bands and a PAN image. Landsat ETM+ is the second dataset that contains eight bands with different resolutions; the bands 1 to 5 and 7 have 30m spatial resolution, band 6 owns $60 \mathrm{~m}$ resolution and band 8 is considered as PAN data with $15 \mathrm{~m}$ resolution. The interval of variation for the $\mathrm{g}_{\mathrm{i}}$ is the same for two datasets and it lies in the interval $[-2,2]$. This range is considered in order to have a wide selection space; however the choice of a larger interval makes the optimization process slower. The number of initial population and the number of bits devoted to each variable are the same for both of two datasets and it is equal to 50 and 10, respectively. The number of iterations for Landsat ETM+ is 100 and it is equal to 200 for IKONOS.

Before we present the simulation results, five evaluation criteria for quantitative comparison of fusion methods are introduced.

\subsection{Spectral and Spatial Quality Indicators}

5.1.1 SAM: The spectral angle mapper (SAM) between two vectors $\boldsymbol{v}$ and $\hat{\boldsymbol{v}}$ is defined as Eq.(23). SAM degree is obtained through averaging over all pixels and it is used as spectral distortion measurement. Zero value of SAM indicates the absence of the spectral distortion [28].

$$
\operatorname{SAM}(\boldsymbol{v}, \hat{\boldsymbol{v}})=\cos ^{-1}\left(\frac{\langle\boldsymbol{v}, \hat{\boldsymbol{v}}\rangle}{\|\boldsymbol{v}\|_{2}\|\hat{\boldsymbol{v}}\|_{2}}\right)
$$

Where $\langle$.$\rangle and \|\cdot\|_{2}$ are inner product and norm operator, respectively. Furthermore, $v$ represents the MS pixel vector and $\hat{v}$ is the distorted vector obtained by applying a fusion method on the MS images.

5.1.2 ERGAS: The "erreur relative globale adimensionnelle de synthèse" (ERGAS) index (or relative global dimensional synthesis error) is defined as follows:

$$
E R G A S=100 \frac{h}{l} \sqrt{\frac{1}{N} \sum_{i=1}^{N}\left(\frac{R M S E^{2}\left(\boldsymbol{B}_{i}\right)}{M_{i}^{2}}\right)}
$$

Where $h$ and $l$ are the resolutions of the PAN and MS images, respectively, $M$ is the mean radiance of the $\mathrm{N}$ spectral bands involved in the fusion [14], and RMSE is the root mean square error computed as follows.

$$
\operatorname{RMSE}^{2}\left(\boldsymbol{B}_{\mathbf{i}}\right)=\operatorname{bias}^{2}\left(\boldsymbol{B}_{\mathbf{i}}\right)+S D^{2}\left(\boldsymbol{B}_{\mathbf{i}}\right)
$$

Where the bias refers to the difference in radiance between the mean of the original image and that of the fused image, and the SD, the standard deviation of the difference image related to the mean of the original image, indicates the level of errors at any pixel. 
5.1.3 Q4 quality index: The universal image quality index (UIQI) has been defined by [29] as follows.

$$
Q(\tilde{\boldsymbol{B}}, \hat{\boldsymbol{B}})=\frac{\sigma_{\tilde{\mathbf{B}} \hat{\mathbf{B}}}}{\sigma_{\tilde{\mathbf{B}}} \sigma_{\hat{\mathbf{B}}}} \cdot \frac{2 \mu_{\tilde{\mathbf{B}}} \mu_{\hat{\mathbf{B}}}}{\left(\mu_{\tilde{\mathbf{B}}}\right)^{2}+\left(\mu_{\hat{\mathbf{B}}}\right)^{2}} \cdot \frac{2 \sigma_{\tilde{\mathbf{B}}} \sigma_{\hat{\mathbf{B}}}}{\sigma_{\tilde{\mathbf{B}}}^{2}+\sigma_{\hat{\mathbf{B}}}^{2}}
$$

Where $\sigma$ is the standard deviation and $\mu$ is the mean of image.

This index includes three terms; the first one is the correlation coefficient between MS image $(\tilde{\mathbf{B}})$ and fused image $(\hat{\mathbf{B}})$, the second and third ones calculate the radiometric distortion and contrast distortion between them. In order to define a unique score index for four-band MS images, Alparone et.al. have proposed Q4 quality index [30]. In this index $\tilde{\mathbf{B}}$ and $\hat{\mathbf{B}}$ in UIQI are replaced with two quaternions $\mathbf{Z}_{\mathbf{1}}$ and $\mathbf{Z}_{\mathbf{2}}$ [28].

5.1.4 Quality with No Reference (QNR): QNR index [28] is determined by calculating the difference of UIQI value for inter-MS band and inter-fused band as spectral distortion and difference of UIQI value between each MS band and the PAN image at low resolution and in the high resolution as spatial distortion. The spectral, $D_{\lambda}$, and spatial distortion, $D_{s}$, can be represented as follows.

$$
\begin{aligned}
& D_{\lambda} \square \sqrt{\frac{1}{L(L-1)} \sum_{l=1}^{L} \sum_{r=1}^{L}\left|Q\left(\hat{\boldsymbol{B}}_{l}, \hat{\boldsymbol{B}}_{\boldsymbol{r}}\right)-Q\left(\tilde{\boldsymbol{B}}_{l}, \tilde{\boldsymbol{B}}_{\boldsymbol{r}}\right)\right|^{2}} \quad r \neq l \\
& D_{s} \square \sqrt{\frac{1}{L} \sum_{l=1}^{L}\left|Q\left(\hat{\boldsymbol{B}}_{l}, \boldsymbol{P}\right)-Q\left(\tilde{\boldsymbol{B}}_{l}, \boldsymbol{P}\right)\right|^{2}}
\end{aligned}
$$

Where Q(.,.) is the UIQI value between two argument, $\mathrm{P}$ is the PAN image and $\tilde{\mathbf{B}}$ and $\hat{\mathbf{B}}$ refer to MS and fused images, respectively. L is the number of MS bands.

QNR index, which uses spectral and spatial quality indices, jointly, is defined as Eq.(10). In this paper the value of parameters $\alpha$ and $\beta$ for QNR index are equal to 1 .

The larger values of UIQI, Q4 and QNR and the lower values of $\mathrm{D}_{\mathrm{s}}, \mathrm{D}_{\lambda}, \mathrm{SAM}$ and ERGAS result in better spectral and spatial quality of the fused images.

\subsection{Case 1: IKONOS Dataset Simulation Results}

We want to simulate the pan-sharpening of IKONOS and Landsat ETM+ images by use of proposed method in Section 5.2 and 5.3. For IKONOS dataset the PAN image size is $1024 \times 1024$ pixels and its spatial resolution is $1 \mathrm{~m}$ and the original size of MS bands is $256 \times 256$ pixels with $4 \mathrm{~m}$ resolution. The PAN image is shown in Figure 2(a), while an original true-colour MS image, up-sampled to the size of PAN image, is shown in Figure $2(b)$.

The MS and PAN images were geometrically registered using the bicubic interpolation algorithm [31]. Then Improved Adaptive IHS-ATW fusion method was applied on IKONOS dataset. Applying the algorithm involved these steps; the IHS transform has been applied to Red, Green, and Blue bands of MS images, and then ATW decomposition of PAN image was done. The intensity component of IHS results and the optimized PAN details using GA and TLA have been reconstructed using ATW reconstruction. This new intensity component with high spatial resolution of PAN image 
obtained by applying our method has been then injected into MS images through the inverse IHS transform. In order to evaluate the superiority of our proposed method, we compare it with three methods introduced in[28] as top ranked fusion algorithms, i.e. GIHS-GA, AWLP and GLP-SDM. First, the fused images are visually compared. The best evaluation for spatial quality of resulted images is visual test. The edges and boundary, blurring and other details are noticed in this test. In the AWLP method, as shown in Figure 2(c), colours have been pretty well distributed and the fused image has high spectral quality but the image has been blurred. As Figure $2(d)$ shows, the GLPSDM outcome has been well enhanced and it has preserved the spectral characteristics pretty good, while the spatial quality has been admissible. As seen from Figure 2(e), the result of the GIHS-GA method has been spatially enhanced and the details have been separated explicitly but the colours in this method have not been relatively well synthesized as the spectral distortion is high. Improved adaptive IHS-ATW result is shown in Figures 2(f) and 2(g). By comparing Figures 2(f) and 2(g) with Figure 2(e), it can be observed that the colour distortions of the fused images are lower than that of GIHS-GA, while the spatial quality is suitable enough. Improved adaptive IHS-ATW method has enhanced the MS images with properties of both IHS and àtrous wavelet methods, namely the details including trees, bare zones and road are explicitly obvious and contain true colours. Considering the above discussion, improved adaptive IHS-ATW method has higher visual quality than the others. Moreover, assessments based on objective indicators presented in section 5.1 confirm this claim (see Table 2).

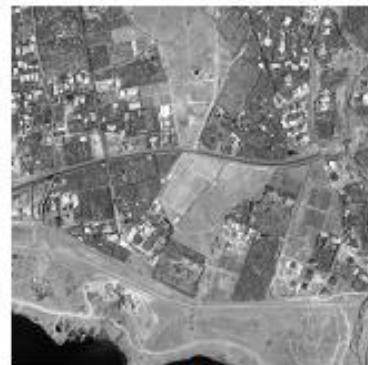

(a)

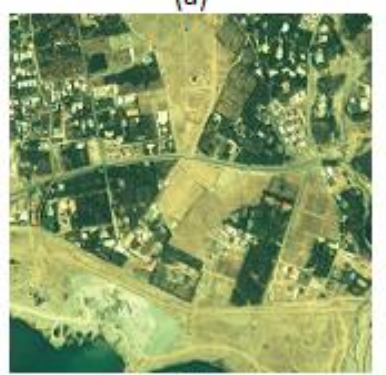

(d)

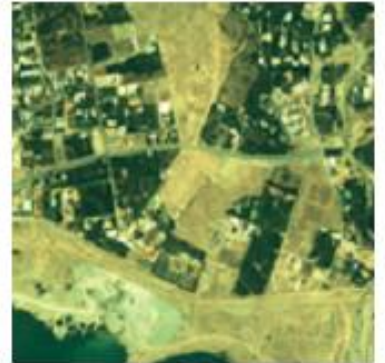

(b)

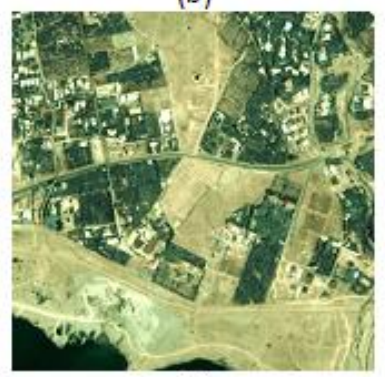

(e)

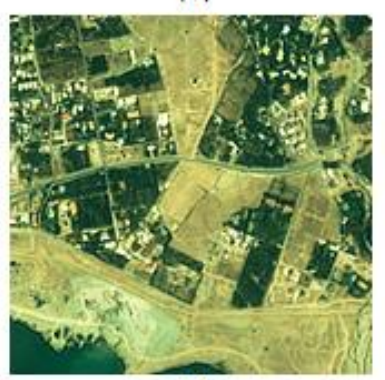

(g)

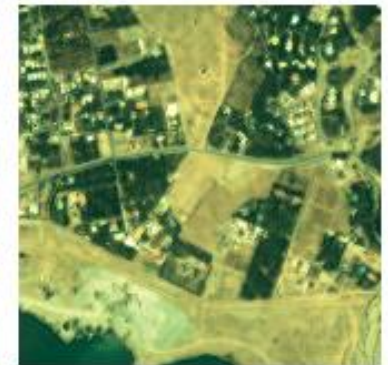

(c)

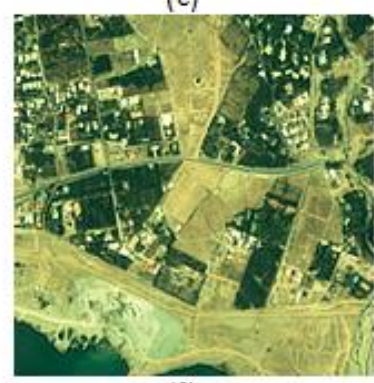

(f)

Figure 2. (a) Pan Image, (b) Resampled MS Image, the Fused Image Obtained by Applying (c) AWLP, (d) GLP-SDM, (e) GIHS-GA, (f) Improved Adaptive IHS-ATW with GA based Optimization and (g) Improved Adaptive IHS-ATW with TLBO (our Proposed Methods) 
Table 1. Quality Evaluation of Fused Images for IKONOS Dataset

\begin{tabular}{|c|c|c|c|c|c|}
\hline Methods & $\mathbf{Q 4}$ & SAM $^{\mathbf{0}}$ & Ds & D $\lambda$ & QNR \\
\hline AWLP & 0.9535 & 1.167 & 0.0857 & 0.1722 & 0.7568 \\
\hline GLP-SDM & 0.9024 & 1.359 & 0.1332 & 0.2335 & 0.6644 \\
\hline GIHS-GA & 0.8941 & 4.15 & 0.1881 & 0.2025 & 0.6474 \\
\hline $\begin{array}{c}\text { Improved adaptive } \\
\text { IHS-ATW using } \\
\text { genetic algorithm }\end{array}$ & 0.9618 & 1.49 & 0.053 & 0.0461 & 0.9033 \\
\hline $\begin{array}{c}\text { Improved adaptive } \\
\text { IHS-ATW using } \\
\text { TLBO algorithm }\end{array}$ & 0.9621 & 1.484 & 0.0528 & 0.0462 & 0.9034 \\
\hline
\end{tabular}

Table 2. Comparison of Methods in Each Objective Indicator for IKONOS Dataset

\begin{tabular}{|c|c|c|c|c|}
\hline Q4 & SAM & $\mathrm{D}_{\mathrm{s}}$ & $\mathbf{D}_{\lambda}$ & QNR \\
\hline $\begin{array}{c}\text { Improved } \\
\text { adaptive } \\
\text { IHS-ATW } \\
\text { using TLBO }\end{array}$ & AWLP & $\begin{array}{c}\text { Improved } \\
\text { adaptive } \\
\text { IHS-ATW } \\
\text { using TLBO }\end{array}$ & $\begin{array}{l}\text { Improved } \\
\text { adaptive } \\
\text { IHS-ATW } \\
\text { using GA }\end{array}$ & $\begin{array}{l}\text { Improved } \\
\text { adaptive } \\
\text { IHS-ATW } \\
\text { using TLBO }\end{array}$ \\
\hline $\begin{array}{l}\text { Improved } \\
\text { adaptive } \\
\text { IHS-ATW } \\
\text { using GA }\end{array}$ & GLP-SDM & $\begin{array}{l}\text { Improved } \\
\text { adaptive } \\
\text { IHS-ATW } \\
\text { using GA }\end{array}$ & $\begin{array}{l}\text { Improved } \\
\text { adaptive } \\
\text { IHS-ATW } \\
\text { using TLBO }\end{array}$ & $\begin{array}{l}\text { Improved } \\
\text { adaptive } \\
\text { IHS-ATW } \\
\text { using GA }\end{array}$ \\
\hline AWLP & $\begin{array}{c}\text { Improved } \\
\text { adaptive } \\
\text { IHS-ATW } \\
\text { using TLBO }\end{array}$ & AWLP & AWLP & AWLP \\
\hline GLP-SDM & $\begin{array}{l}\text { Improved } \\
\text { adaptive } \\
\text { IHS-ATW } \\
\text { using GA }\end{array}$ & GLP-SDM & GIHS-GA & GLP-SDM \\
\hline GIHS-GA & GIHS-GA & GIHS-GA & GLP-SDM & GIHS-GA \\
\hline
\end{tabular}

As can be seen in table 1, the quantitative values obtained by applying our method are all far better than the other methods. For a better comparison, table 2 shows the superiority order of all methods, namely for each index the methods have been ordered from top of column for best quality to the bottom of column for worst quality of that index.

\subsection{Case 2: Landsat ETM+ Dataset Simulation Results}

The second dataset employed for testing the mentioned methods is Landsat ETM+. The PAN image size considered for this experiment is also $512 \times 512$ pixels (see Figure $3(a)$ ) and the original size of bands $1-5$ and 7 is $256 \times 256$ pixels and it is $128 \times 128$ pixels for band 6. All of the MS images have been up-sampled to the scale of PAN image; see false colour composition of band 4, 5, and 3 in Figure 3(b). By applying the steps presented in previous section, the results for each method have been obtained and shown in Figure 3.

As seen from Figures 3(c), for the AWLP, there is low spatial quality in the result, and the image resulted through this method has been blurred. As illustrated in figure $3(d)$, the result of GLP-SDM method owns well spectral quality but it has been a little blurred too. 
For the GIHS-GA fusion method, Figure 3(e), the spatial information has been appropriately injected to the image, and the details have been explicitly separated. The reason of its high values obtained for $S A M$ and $D_{\lambda}$ index value may be due to the fact that the colours in some areas become dark. As can be seen from Figures $3(f)$ and $3(g)$, the result of the improved adaptive IHS-ATW with GA and TLA optimization methods show high spectral quality, besides the details on the fused images are obviously revealed. Table 3 shows better spectral and spatial quality assessments in the results of improved adaptive IHS-ATW methods with respect to the other methods.

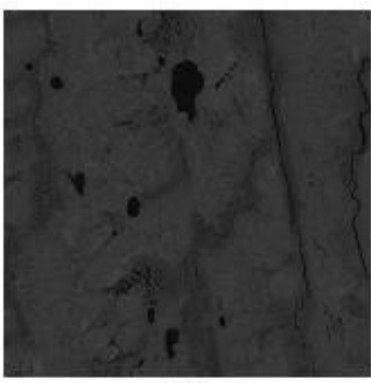

(a)

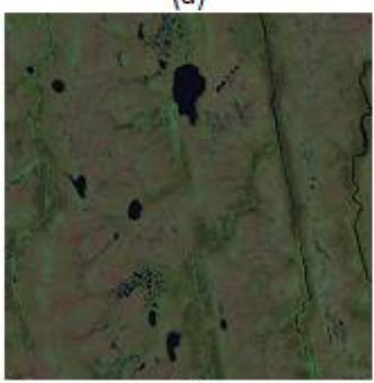

(d)

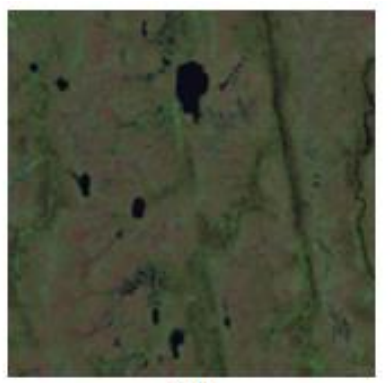

(b)

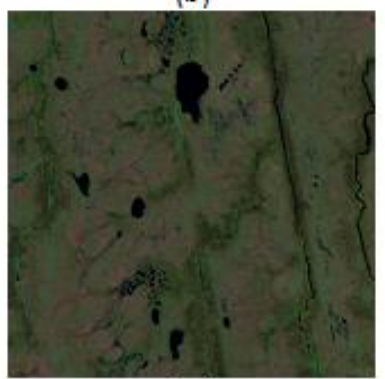

(e)

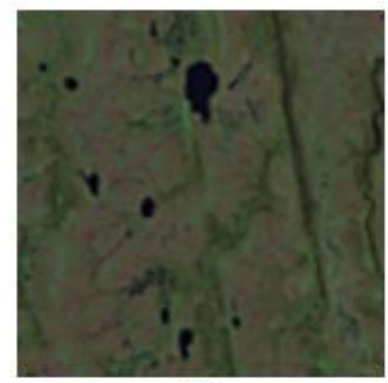

(c)

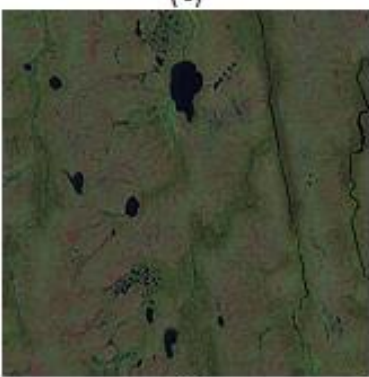

(f)

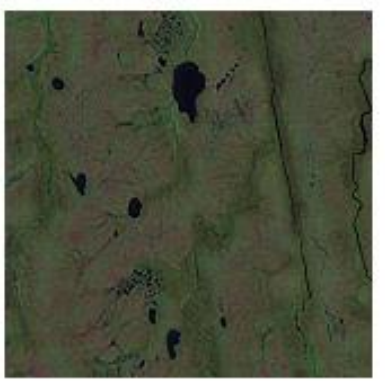

(g)

Figure 3. The Fusion Results of Landsat ETM+ Images using Different Methods. (a) Pan Image, (b) False Colour Resampled MS Image, the Fused Image Obtained by Applying (c) AWLP, (d) GLP-SDM, (e) GIHS-GA, (f) Improved Adaptive IHS-ATW with GA based Optimization and ( $g$ )Improved Adaptive IHS-ATW with TLBO (our Proposed Methods). Note that Bands 4, 5 , and 3 were Combined as a Colour Image 
Table 3. Quality Evaluation of Fused Images for Landsat ETM+ Dataset

\begin{tabular}{|c|c|c|c|c|c|}
\hline Methods & Q4 & $\begin{array}{c}\text { SA } \\
\mathbf{M}^{\mathbf{0}}\end{array}$ & $D_{s}$ & $\overline{\mathbf{D}_{\lambda}}$ & $\begin{array}{l}\text { QN } \\
\mathbf{R}\end{array}$ \\
\hline AWLP & 0.98 & 1.9 & 0.112 & $\begin{array}{l}0.12 \\
2\end{array}$ & 0.78 \\
\hline GLP-SDM & $\begin{array}{l}0.96 \\
1\end{array}$ & 5.02 & 0.163 & $\begin{array}{l}0.14 \\
6\end{array}$ & $\begin{array}{l}0.71 \\
5\end{array}$ \\
\hline GIHS-GA & $\begin{array}{l}0.96 \\
2\end{array}$ & 3.17 & 0.242 & $\begin{array}{l}0.14 \\
5\end{array}$ & $\begin{array}{l}0.64 \\
8\end{array}$ \\
\hline $\begin{array}{l}\text { Improved adaptive } \\
\text { IHS-ATW using } \\
\text { genetic algorithm }\end{array}$ & $\begin{array}{l}0.99 \\
1\end{array}$ & 1.69 & $\begin{array}{c}0.113 \\
3\end{array}$ & $\begin{array}{l}0.10 \\
8\end{array}$ & $\begin{array}{l}0.79 \\
1\end{array}$ \\
\hline $\begin{array}{l}\text { Improved adaptive } \\
\text { IHS-ATW using } \\
\text { TLBO algorithm }\end{array}$ & $\begin{array}{l}0.99 \\
1\end{array}$ & 1.74 & $\begin{array}{c}0.108 \\
3\end{array}$ & $\begin{array}{l}0.10 \\
35\end{array}$ & $\begin{array}{l}0.79 \\
9\end{array}$ \\
\hline
\end{tabular}

\section{Conclusion}

In this paper we concluded that the IHS pan-sharpening method provides high spatial quality in the fused images, however, the spectral quality of the results is unacceptable. We, therefore, proposed a new IHS-based pan-sharpening method established upon using adaptive ATW decomposition based injection to maintain spectral properties of MS images. In our injection model, the gains of detail images have been optimized to obtain maximum spatial and spectral quality via GA and TLA. The simulation results have confirmed the superiority of these new methods. Nevertheless, these methods are somehow time consuming which will be the subject of our future works.

\section{References}

[1] C. Pohl and J. L. van Gendern, "Multisensor image fusion in remote sensing: concepts, methods and applications", International Journal of Remote Sensing., vol. 19, (1998), pp. 823-854.

[2] L. Alparone, L. Wald, J. Chanussot, C. Thomas, P. Gamba, and L. M. Bruce, "Comparison of pansharpening algorithms: Outcome of the 2006 GRS-S data-fusion contest", IEEE Transactions on Geoscience and Remote Sensing., vol. 45, (2007), pp. 3012-3021.

[3] B. Aiazzi, S. Baronti, and M. Selva, "Improving component substitution pansharpening through multivariate regression of MS+ Pan data", IEEE Transactions on Geoscience and Remote Sensing., vol. 45, (2007), pp. 3230-3239.

[4] K. Amolins, Y. Zhang, and P. Dare, "Wavelet based image fusion techniques--An introduction, review and comparison", ISPRS Journal of Photogrammetry and Remote Sensing., vol. 62, (2007), pp. 249-263.

[5] W. Carper, T. Liliesand, and R. Kiefer, "The use of intensity-hue-saturation transformations for merging SPOT panchromatic and multispectral image data", Photogrammetric Engineering and remote sensing., vol. 56, (1990), pp. 459-467.

[6] Z. Wang, D. Ziou, C. Armenakis, D. Li, and Q. Li, "A comparative analysis of image fusion methods", IEEE Transactions on Geoscience and Remote Sensing., vol. 43, (2005), pp. 1391-1402.

[7] P. CHAVEZ, S. C. Sides, and J. A. Anderson, "Comparison of three different methods to merge multiresolution and multispectral data- Landsat TM and SPOT panchromatic", Photogrammetric Engineering and remote sensing., vol. 57, (1991), pp. 295-303.

[8] J. Liu, "Smoothing filter-based intensity modulation: a spectral preserve image fusion technique for improving spatial details", International Journal of Remote Sensing., vol. 21, (2000), pp. 3461-3472.

[9] J. Zhou, D. L. Civco, and J. A. Silander, "A wavelet transform method to merge Landsat TM and SPOT panchromatic data", International Journal of Remote Sensing., vol. 19, (1998), pp. 743-757.

[10] W. Yao and M. Han, "Improved GIHSA for image fusion based on parameter optimization", International Journal of Remote Sensing., vol. 31, (2010), pp. 2717-2728.

[11] S. Rahmani, M. Strait, D. Merkurjev, M. Moeller, and T. Wittman, "An adaptive IHS pan-sharpening method", IEEE Geoscience and Remote Sensing Letters., vol. 7, (2010), pp. 746-750.

[12] T. Te-Ming, S. Shun-Chi, H. Hsuen-Chyun, and P. S. Huang, "A new look at IHS-like image fusion methods", Information fusion, vol. 2, (2001), pp. 177-186. 
[13] Y. Zhang, "A new merging method and its spectral and spatial effects", International Journal of Remote Sensing., vol. 20, (1999), pp. 2003-2014.

[14] M. González-Audícana, J. L. Saleta, R. G. Catalán, and R. García, "Fusion of multispectral and panchromatic images using improved IHS and PCA mergers based on wavelet decomposition", IEEE Transactions on Geoscience and Remote Sensing., vol. 42, (2004), pp. 1291-1299.

[15] B. Aiazzi, L. Alparone, S. Baronti, and A. Garzelli, "Context-driven fusion of high spatial and spectral resolution images based on oversampled multiresolution analysis", IEEE Transactions on Geoscience and Remote Sensing., vol. 40, (2002), pp. 2300-2312.

[16] M. J. Shensa, "The discrete wavelet transform: Wedding the a trous and Mallat algorithms", IEEE Transactions on Signal Processing., vol. 40, (1992), pp. 2464-2482.

[17] Y. Chibani and A. Houacine, "The joint use of IHS transform and redundant wavelet decomposition for fusing multispectral and panchromatic images", International Journal of Remote Sensing., vol. 23, (2002), pp. 3821-3833.

[18] T. Stathaki, "Image fusion: algorithms and applications", Academic Press, (2008).

[19] M. Choi, "A new intensity-hue-saturation fusion approach to image fusion with a tradeoff parameter", IEEE Transactions on Geoscience and Remote Sensing., vol. 44, (2006), pp. 1672-1682.

[20] T. Ranchin, B. Aiazzi, L. Alparone, S. Baronti, and L. Wald, "Image fusion--the ARSIS concept and some successful implementation schemes", ISPRS Journal of Photogrammetry and Remote Sensing., vol. 58, (2003), pp. 4-18.

[21] B. Garguet-Duport, J. Girel, J. M. Chassery, and G. Patou, "The use of multiresolution analysis and wavelets transform for merging SPOT panchromatic and multispectral image data", Photogrammetric Engineering and remote sensing., vol. 62, (1996), pp. 1057-1066.

[22] B. Aiazzi, S. Baronti, F. Lotti, and M. Selva, "A comparison between global and context-adaptive pansharpening of multispectral images", IEEE Geoscience and Remote Sensing Letters., vol. 6, (2009), pp. 302-306.

[23] A. Golibagh Mahyari and M. Yazdi, "Panchromatic and Multispectral Image Fusion Based on Maximization of Both Spectral and Spatial Similarities", IEEE Transactions on Geoscience and Remote Sensing., vol. 49, (2011), pp. 1976-1985.

[24] X. Otazu, González-Audícana, M., Fors, O., Núñez, J., "Introduction of sensor spectral response into image fusion methods. Application to wavelet-based methods", IEEE Transactions on Geoscience and Remote Sensing., vol. 43, (2005), pp. 2376-2375.

[25] B. Aiazzi, L. Alparone, S. Baronti, and M. Selva, "Generalised Laplacian pyramid-based fusion of MS+ $\mathrm{P}$ image data with spectral distortion minimisation", International Archives of photogrammetry and Remote Sensing., (2002).

[26] S. Sivanandam and S. Deepa, "Introduction to genetic algorithms", Springer Verlag, (2007).

[27] Z. Michalewicz, "Genetic algorithms+ data structures", Springer, (1996).

[28] L. Alparone, B. Aiazzi, S. Baronti, A. Garzelli, F. Nencini, and M. Selva, "Multispectral and panchromatic data fusion assessment without reference", Photogrammetric Engineering and Remote Sensing., vol. 74, (2008), p. 193.

[29] Z. Wang and A. C. Bovik, "A universal image quality index", IEEE Signal Processing Letters., vol. 9, (2002), pp. 81-84

[30] L. Alparone, S. Baronti, A. Garzelli, and F. Nencini, "A global quality measurement of pan-sharpened multispectral imagery", IEEE Geoscience and Remote Sensing Letters., vol. 1, (2004), pp. 313-317.

[31] R. G. Keys, "Cubic convolution interpolation for digital image processing", IEEE Transactions on Acoustics, Speech and Signal Processing., vol. 29, (1981), pp. 1153-1160. 
International Journal of Signal Processing, Image Processing and Pattern Recognition Vol.9, No.3 (2016) 